\title{
The Role of MRI Scanning in Neuro-Ophthalmology
}

\author{
D. J. SPALTON ${ }^{1}$ and K. A. TONGE ${ }^{2}$ \\ London
}

\begin{abstract}
Summary
The role of high resolution magnetic resonance imaging (MRI) in the assessment of optic atrophy, chiasmal compression, and posterior fossa lesions is demonstrated. Good spatial resolution, absence of bony artifact and multiplanar imaging are significant advantages over CT scanning in these areas, as is the ability to detect areas of demyelination. Orbital MRI offers future potential but at present we think that $\mathrm{CT}$ scanning provides better spatial resolution and is more cost effective for the orbit.
\end{abstract}

Whilst almost any part of the brain can produce lesions of neuro-ophthalmic interest the neuro ophthalmologist is most commonly faced with the investigation of space occupying lesions in the region of the chiasm, the posterior fossa with ocular motility disorders, and the investigation of undiagnosed optic atrophy or lesions of the orbit. There is a need to rationalise the use of CT and magnetic resonance imaging (MRI) in the imaging of these areas to facilitate the diagnosis, reduce harmful investigation and save expense. At St. Thomas' Hospital we have had access to a high field MRI scanner (1.5 Tesla) and a Siemens DRH CT scanner for two to three years and this paper reviews the relative merits of both techniques.

In contrast to other imaging techniques such as CT scanning or ultrasound which measure simple parameters to construct the image (tissue radiodensity or interface reflections), the MRI signal is a composite signal reflecting the concentration and environment of protons $(\mathrm{H}+$ ions $)$ in the tissues. Depending on the parameters used to produce the MR image either the proton density (T1 weighted image) or some of the magnetic properties of the tissue in which the protons lie (T2 weighted image) can be depicted. The basis of MR scanning is that each tissue has characteristic $\mathrm{T} 1$ and $\mathrm{T} 2$ times which are altered by disease. Fat produces a high signal because of its high $\mathrm{H}+$ ion concentration. In other tissues the clinical image is largely influenced by the amount of intra and extracellular water present: those protons in structures such as bone, protein or myelin are more tightly bound and do not contribute greatly to the MRI image. The T1 image can be thought of as reflecting the amount of tissue water present and the T2 image the environment of the water (i.e. the size of other molecules, their ionic state, and the lattice structure).

The MRI images are obtained by reorientating the protons by a radio frequency pulse to each other within a strong magnetic field and measuring the radio waves released by their return to their original orientation within the field. The strength and geometrical relationship of the magnetic field and radio-frequency pulse influence the type of image obtained. In most clinical work a 'spin echo' sequence is used although on occasions an inversion recovery' sequence can have advantages (other sequences such as saturation recovery are not commonly used). By altering the time

From: Departments of Ophthalmology ${ }^{1}$ and Radiology ${ }^{2}$ St. Thomas' Hospital, London SE1 7EH.

Correspondence to: Mr D. J. Spalton, Medical Eye Unit, St. Thomas' Hospital, Lambeth Palace Road, London SE1 7EH. 


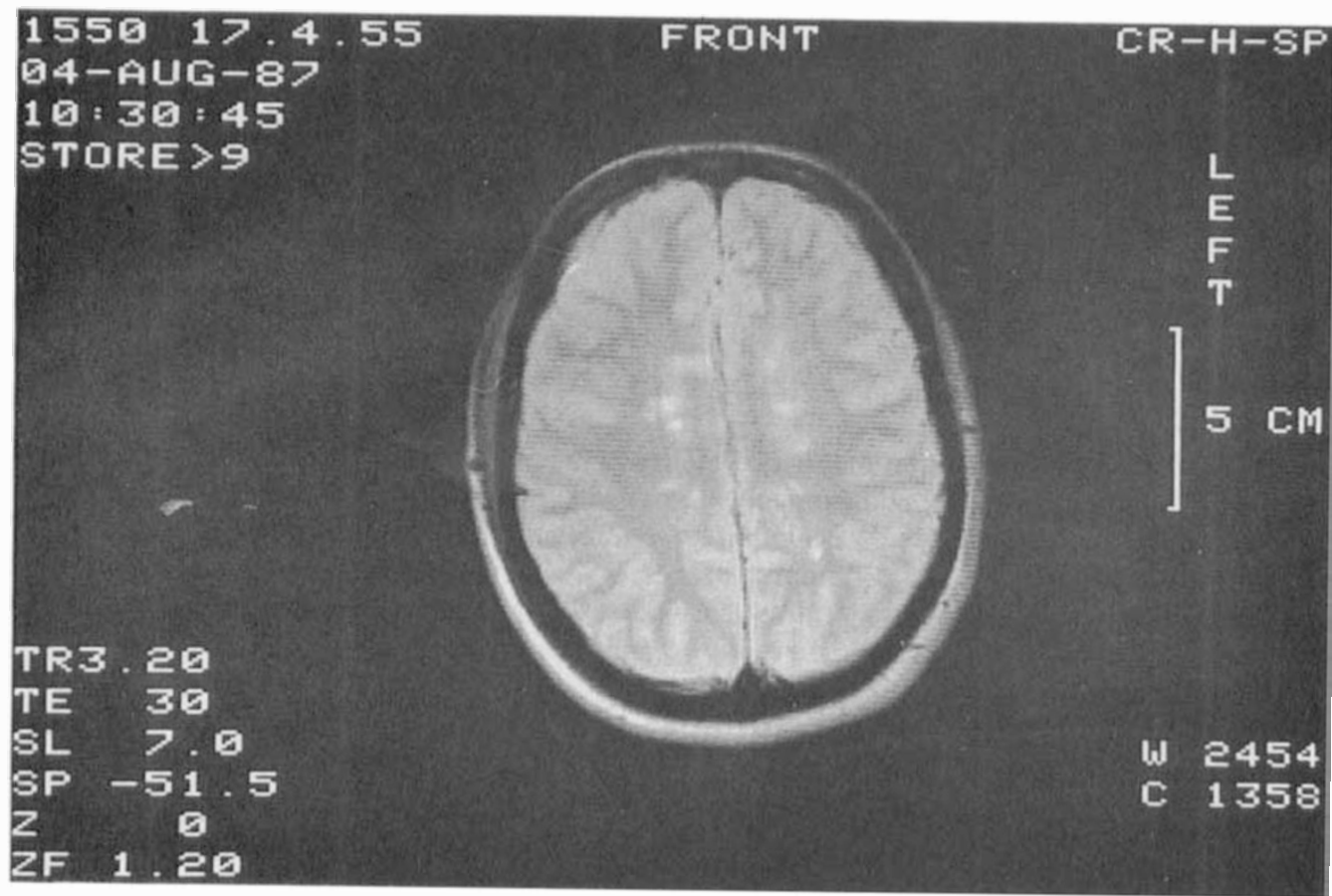

Fig. 1 a

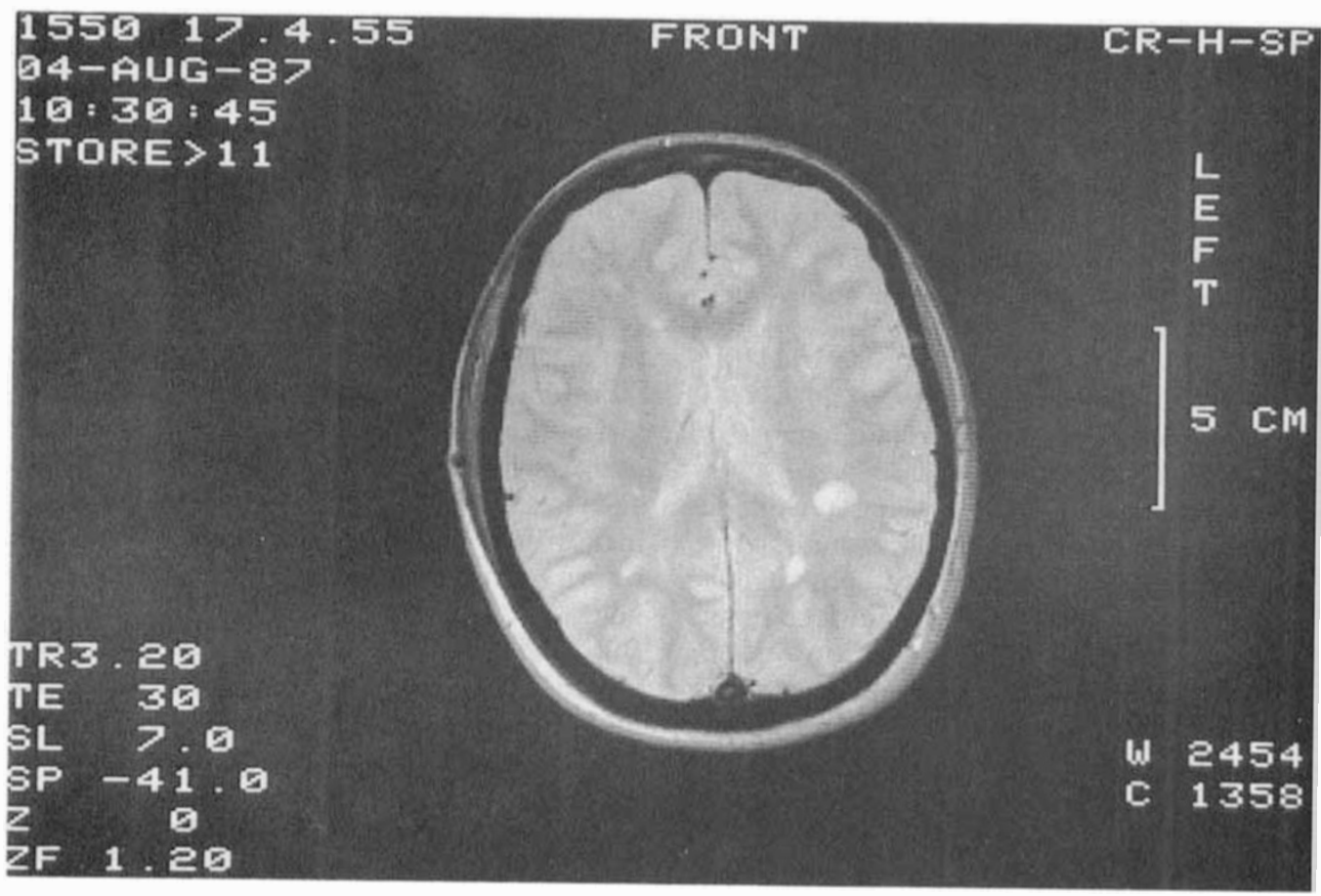

Fig. 1 b

Fig. 1a and b. Case I. T2 weighted axial scans show numerous high intensity lesions in the cerebral hemisphere white matter compatible with demyelination. 


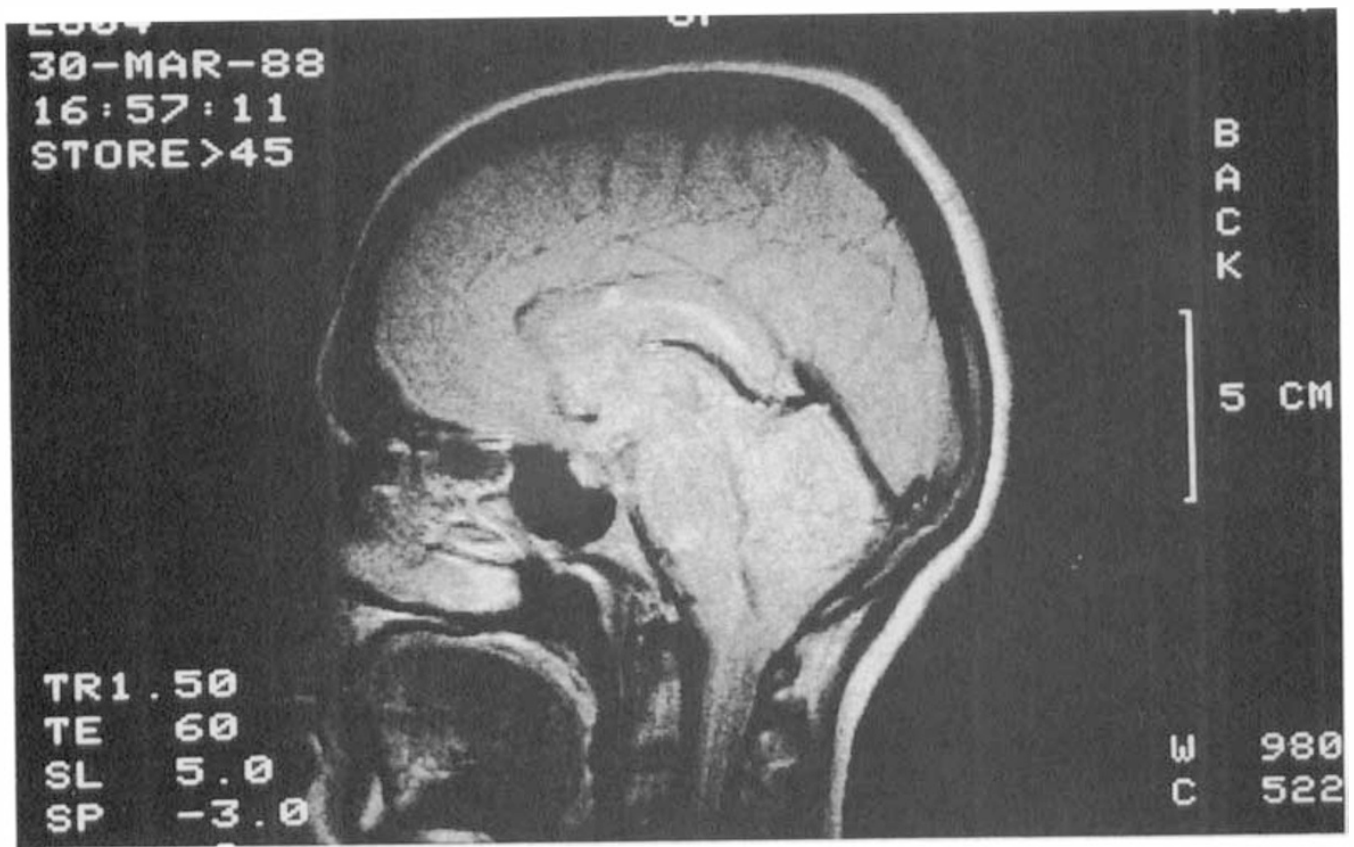

Fig. 2. T2 weighted scan showing high intensity signals in the pons and corpus callosum in a patient with systemic vasculitis. These lesions can be confused with those of Fig. 1.

between radio frequency pulses (repetition time) or the time of sampling the emitted signals (echo time) the operator can weight the image to either the $\mathrm{T} 1$ or $\mathrm{T} 2$ parameters. The ability to alter so many parameters of the MRI image greatly adds to the complexity of the technique but also offers great versatility as some lesions are shown much more easily by one or other parameter. In clinical practice, though, set routines are used depending on the region to be imaged and the clinical question to be answered.

Advantages of MRI over CT scanning are that no ionising radiation is used and images can be constructed in any plane. The absolute contraindications to MRI are cardiac pacing and intraocular magnetic foreign bodies. Other relative contraindications are clipped intracranial aneurysms (depending on the type of clip used), the need to use non-magnetic equipment for examination under anaesthesia and the possibility of producing localised heating effects. The latter is largely theoretical although conceivably it could be important to the ocular lens.

The images shown in this report are taken using a 1.5 Tesla scanner. This is one of the highest field strengths at present in clinical use and gives greatly superior anatomical detail to previous lower power instruments. There are two reasons to perform neuroradiological imaging: the first is to anatomically localise a lesion and the second is to attempt a tissue diagnosis. From the clinical point of view T1 weighted images produce a picture similar to an air encephalogram giving better anatomical detail than the $\mathrm{T} 2$ image but $\mathrm{T} 2$ images are helpful in making a tissue diagnosis in that they provide better contrast between the normal and abnormal (e.g. lesions compatible with demyelination have an enhanced T2 signal).

\section{Illustrative Cases}

\section{Optic Atrophy}

Case one, presented with bilateral optic atrophy and imaging was required to exclude a compressive lesion. The MRI scan excluded this, using $\mathrm{T} 1$ weighting but also showed multiple high signal lesions on the T2 weighted scan suggestive of demyelination (Figs. 1a, b). Typically, these lesions are located adjacent to the ventricles in the cerebral white matter, pons or cerebellum. Whilst 


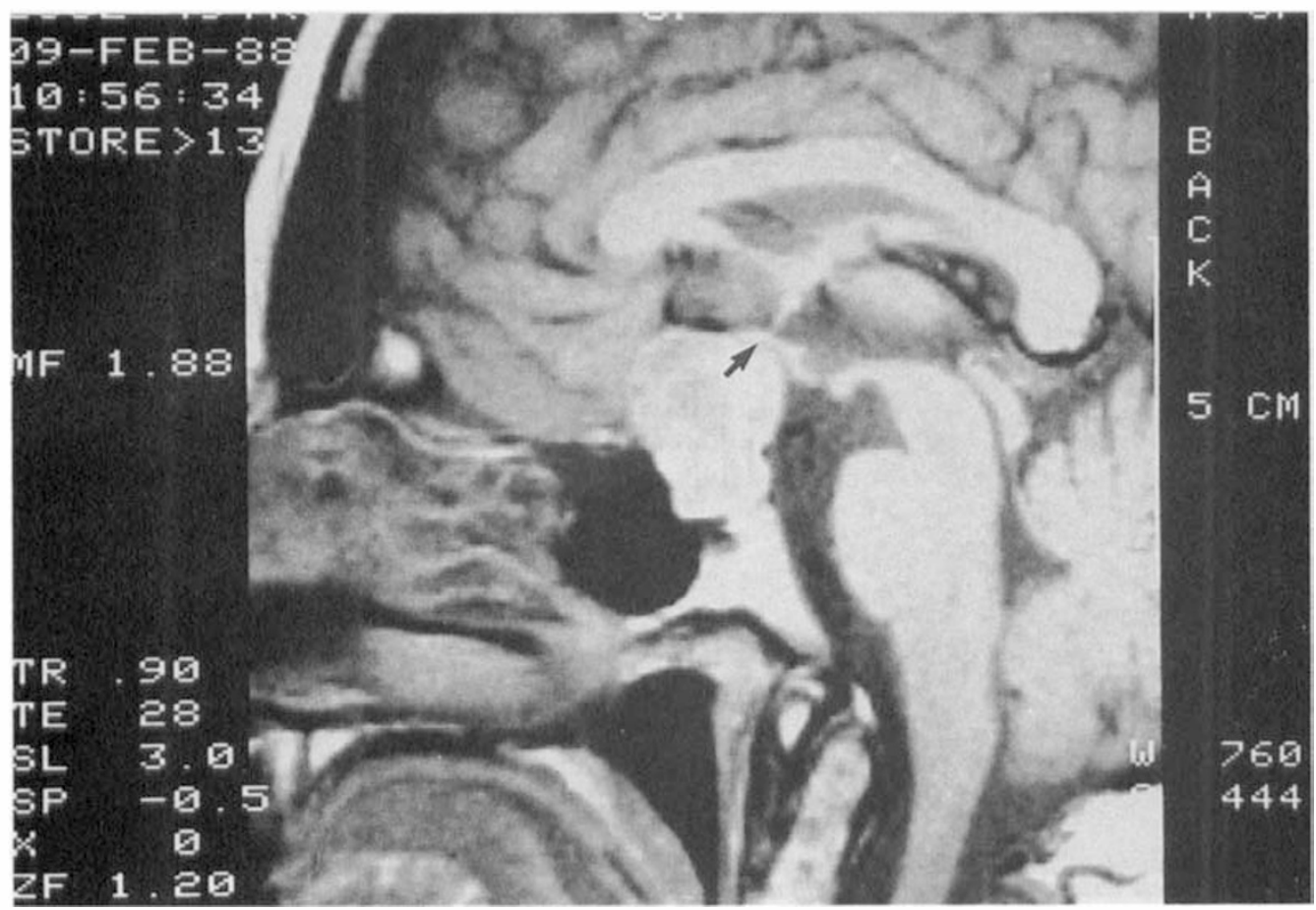

Fig. 3a

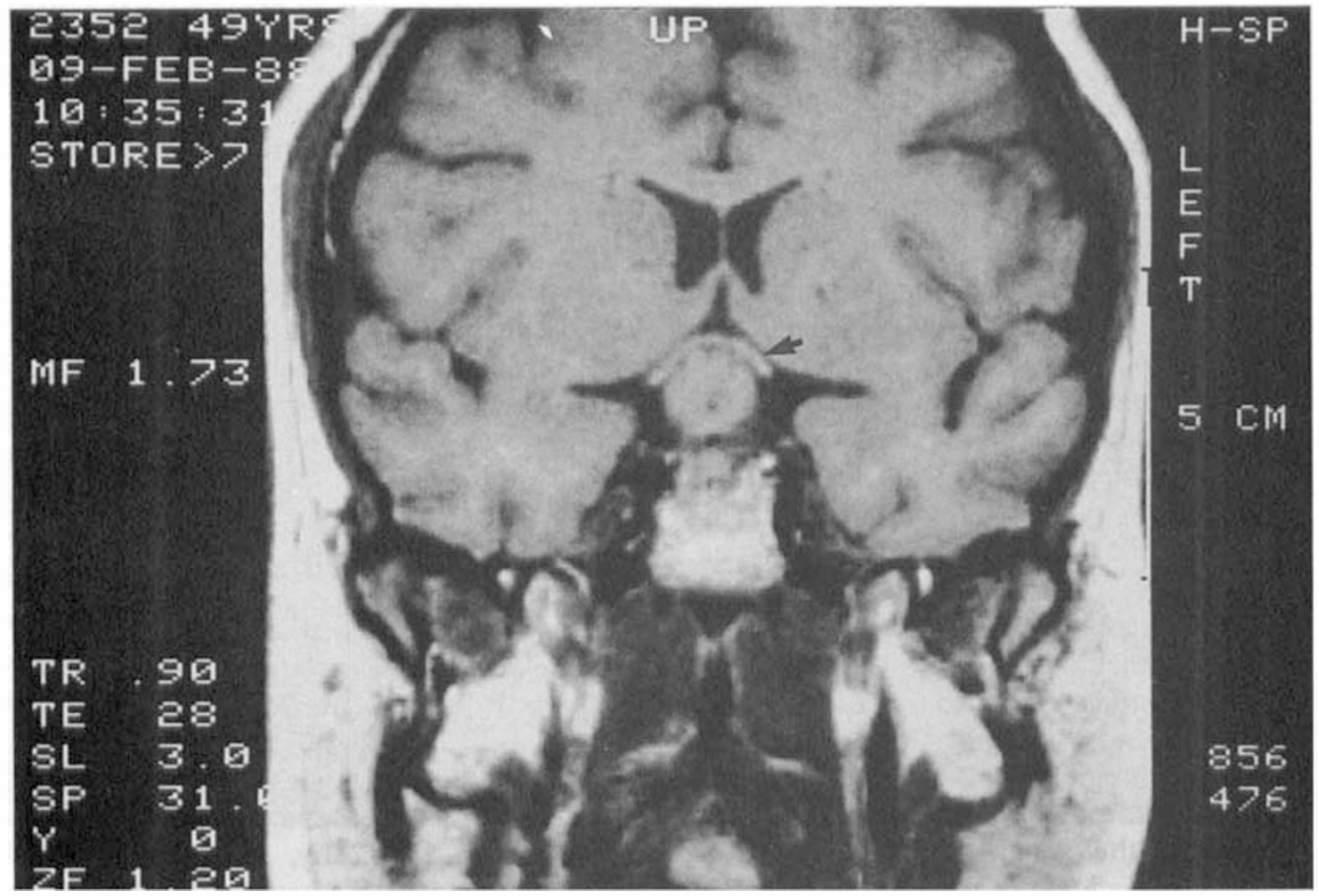

Fig. 3b 


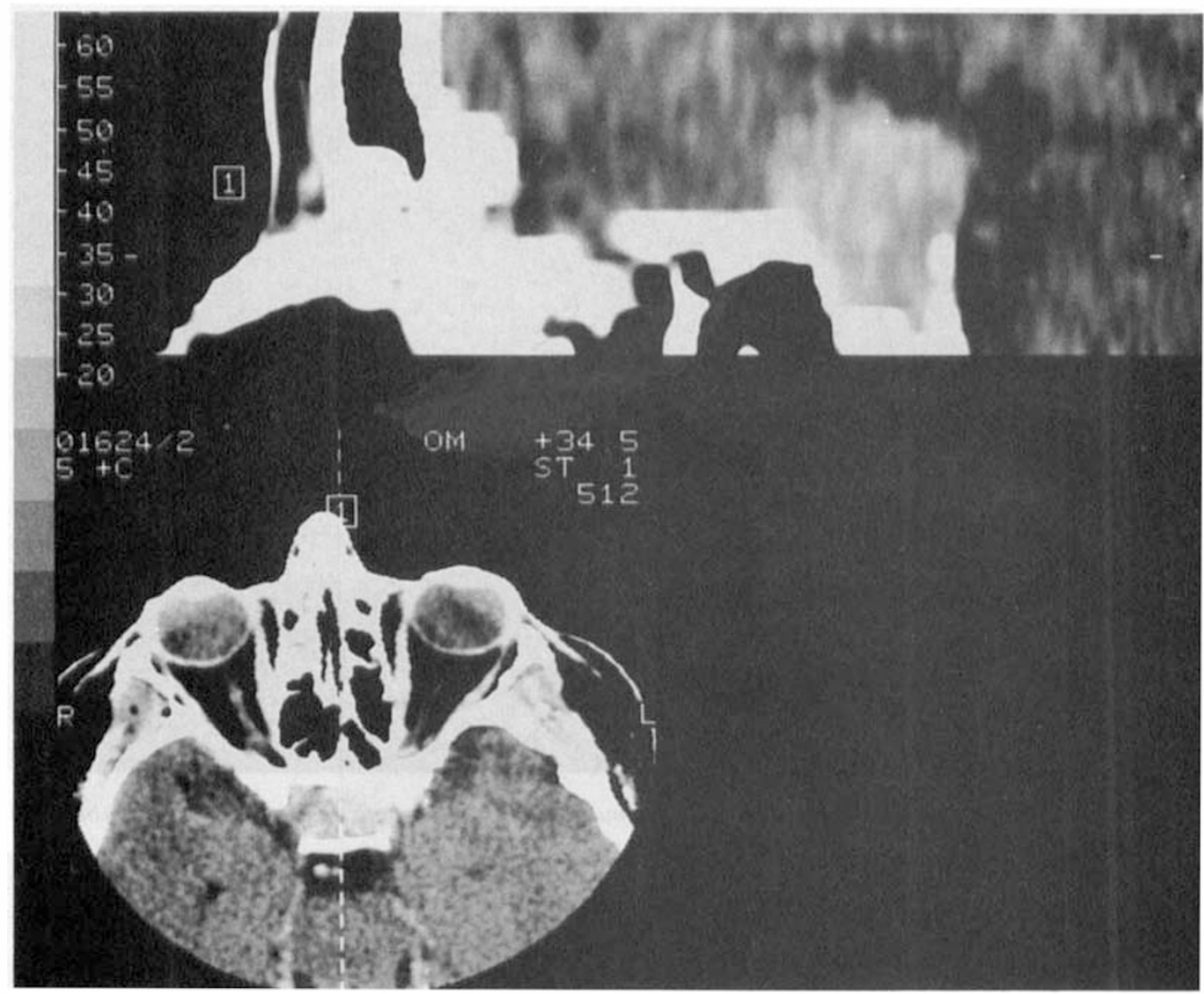

Fig. 3c

Fig. 3a, b and c. MRI T1 weighted scans show the chiasm (arrow) stretched over a pituitary adenoma $(A, B)$. Figure C shows the CT scan of the same patient. Note the better anatomical detail of the MRI scans.

these are suggestive of demyelination and useful confirmatory evidence of a diagnosis of multiple sclerosis, they are not in themselves diagnostic as similar lesions can be seen with autoimmune vasculitis (Fig. 2), multiple infarcts, post-viral infections and occasionally similar isolated lesions can be seen in apparently normal brains.

\section{Chiasmal Lesions}

MRI scanning gives excellent anatomical localisation of pituitary tumours and details of the relationship to the chiasm (Fig. 3) and cavernous sinus can be seen (Fig. 4). Case 2 presented with progressive visual failure following previous surgery for a cystic craniopharyngioma. The CT scan (Fig. 5a) was reported as showing an empty sella but the
MRI (Fig. 5b) clearly shows a large cystic recurrence.

\section{Posterior Fossa Lesions}

MRI has very significant advantages over CT in the management of lesions in this region because of good tissue contrast and the absence of bony artefact (unavoidable and due to beam hardening in CT). Case 3 presented with bilateral IV nerve palsies and a lymphoma. Figure 6 shows a haemorrhage involving the inferior colliculus (haemorrhage and the length of time it has been present can often be deduced with certainty because of the strong magnetic properties of the degradation products of haem). Case 4 presented with a right VI palsy with multiple sclerosis. The causative lesion can be seen (Fig. 7), 


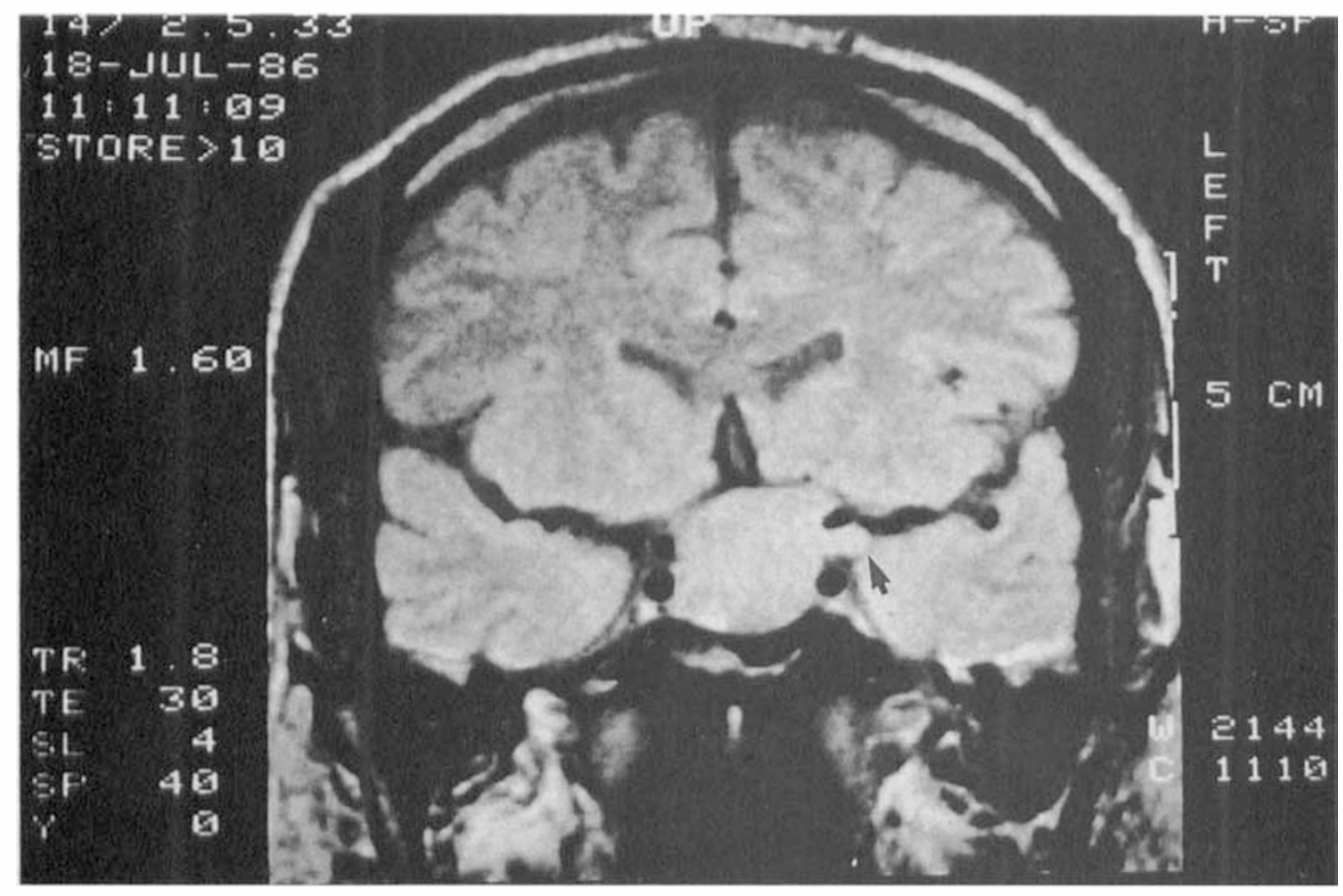

Fig. 4. Coronal MRI T1 scan showing pituitary adenoma invading the cavernous sinus (arrow) on the left between the carotid syphon.

other areas compatible with demyelination were demonstrated on the scan.

\section{Orbit}

Conventional MRI scanning of the orbit is less satisfactory than that of the brain. Calcification, which is so important in defining the orbital boundaries and their involvement in the pathological process, has no signal and is therefore indistinguishable from air in the sinuses. Good images take several minutes to acquire and movement and chemical shift artefact occur and detract from the image. Chemical shift occurs at the boundary between fat and soft tissue only in one coordinate and can be obviated by changing the methods of data collection. It consists of a spurious dark band at the boundary and is worse on $\mathrm{T} 1$ weighted images. Conventional MRI slices are thicker than CT. The latter is usually quicker, more easily interpretable and more cost effective. Better orbital MRI scans can be obtained using radio antenna (surface coils) applied close to the region of interest to collect a stronger signal from it. With different sequences of data collection (Figs. 8a,b,c) better images can be obtained, but these are time consuming (typically 12 minutes) and demand special cooperation from the patient and more technical expertise from the operator. Because the signal of moving protons is lost, providing a negative image, MRI scanning can be particularly useful in demonstrating vascular flow, for example, in distinguishing a carotico-cavernous fistula from a thrombosis in the superior ophthalmic vein. Special techniques have been developed to demonstrate and even measure flow but in general further advances are necessary before MRI replaces $\mathrm{CT}$ as the technique of choice in the orbit.

\section{Discussion}

MRI scanning has the great advantage of not using harmful $\mathrm{x}$-rays and because of the absence of bony artefact, and the facility to image in any plane, it produces excellent anatomical localisation of lesions. This is, in many cases superior to CT scanning. These features are particularly advantageous in the investigation of lesions in the area of the chiasm or the posterior fossa. It has the disad- 


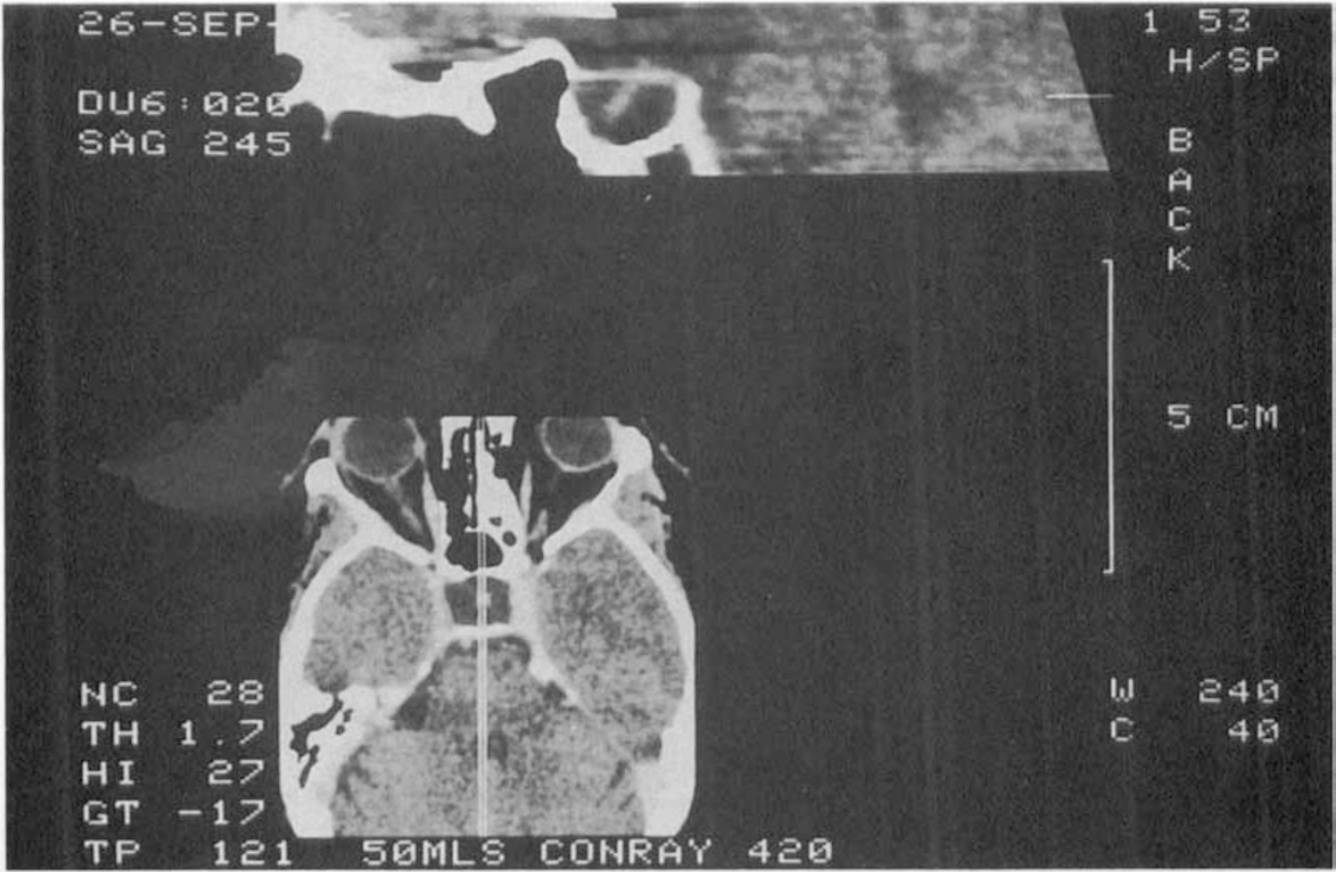

Fig. 5a

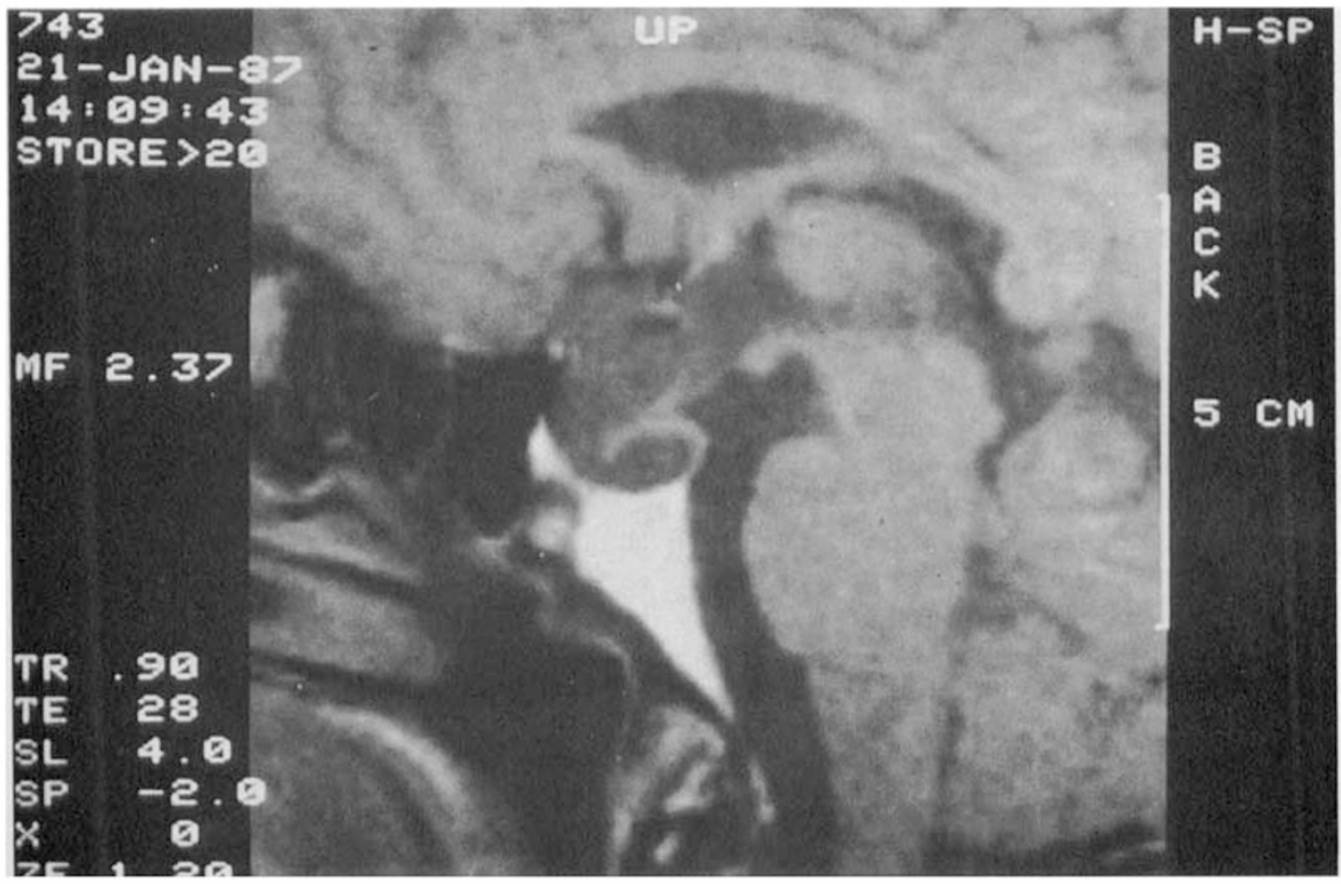

Fig. 5b

Fig. 5a and b. Case 2. A cyst of Rathke's pouch. The CT scan (5a) was reported as showing an empty sella but the $M R I$ scan (5b) demonstrates a large cystic lesion compressing the anterior end of the III ventricle. 


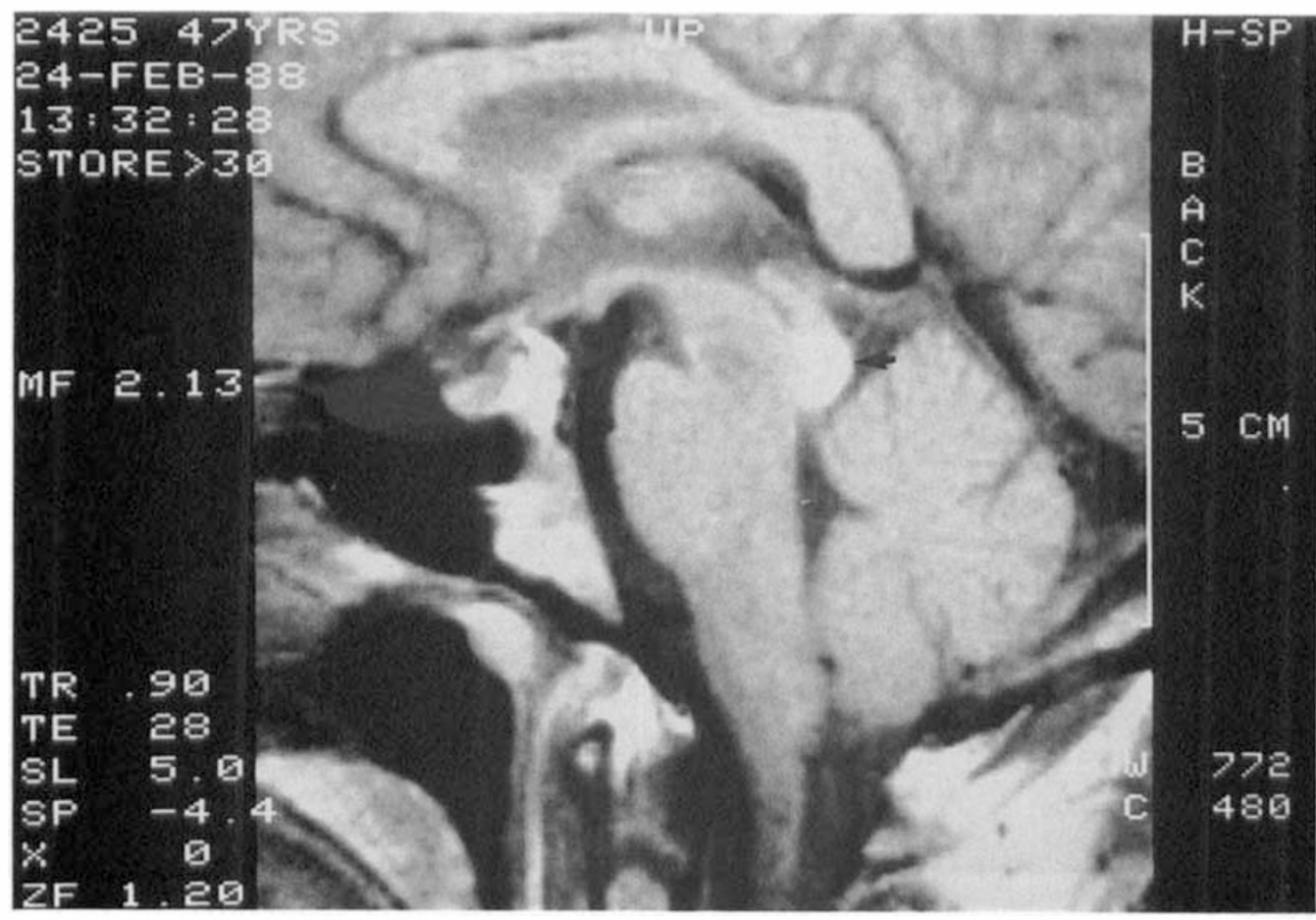

Fig.6. Case 3. This patient presented with bilateral IV nerve palsies and alymphoma. Thesaggital T1 weighted MRI shows a high intensity space occupying lesion in the inferior colliculus, compatible with a haemorrhage (arrow).

vantage that calcification, which is an important feature of so many pathological processes, is not well demonstrated. Other problems are that MRI is prone to movement and chemical shift artefacts and that the technique is complex and requires different expertise to CT scanning. Expense is broadly similar to that of CT scanning.

One of the major advantages of MRI is its superior ability to recognise lesions compatible with demyelination in multiple sclerosis (MS). Typically these subclinical lesions, with a high T2 signal, are found in the periventricular areas and in the white matter of the cerebral hemispheres in the frontal and occipital lobes, the cerebellum and pons. ${ }^{1}$ Lesions in the spinal cord or optic nerves can only be visualised with special techniques because of the small size of the lesion and difficulty of contrasting the optic nerve signal to that of orbital fat. ${ }^{2}$ The abnormal MRI signal is thought to be derived from additional tissue water in areas of astrocytic gliosis to which it is more sensitive than CT scanning. Plaques tend to persist but with time to become less bright and MRI gives the possibility of distinguishing between acute or chronic lesions by observing the difference between $\mathrm{T} 1$ and $\mathrm{T} 2$ signals. $^{3,4}$ A more effective distinction is obtained by the administration of gadolinium. This gives changes in the image similar to those of contrast enhancement in CT which are related to blood perfusion. MS lesions have typical features and distribution on MRI but the appearances can be mimiced by other causes such as vasculitis or infarction so that scans have to be interpreted in the light of the clinical picture. MRI scanning is particularly useful in neuro-ophthalmology in the investigation of optic atrophy where demyelinating disease is suspected, allowing space occupying lesions to be excluded, and providing additional evidence of lesions compatible with demyelination in the brain. Approximately $60 \%$ of patients presenting with acute optic neuritis will show intracranial lesions on MRI scanning. This corresponds approximately to the percentage of patients that can be expected to develop MS over the next 15 years. ${ }^{5}$ It is not known for certain whether 


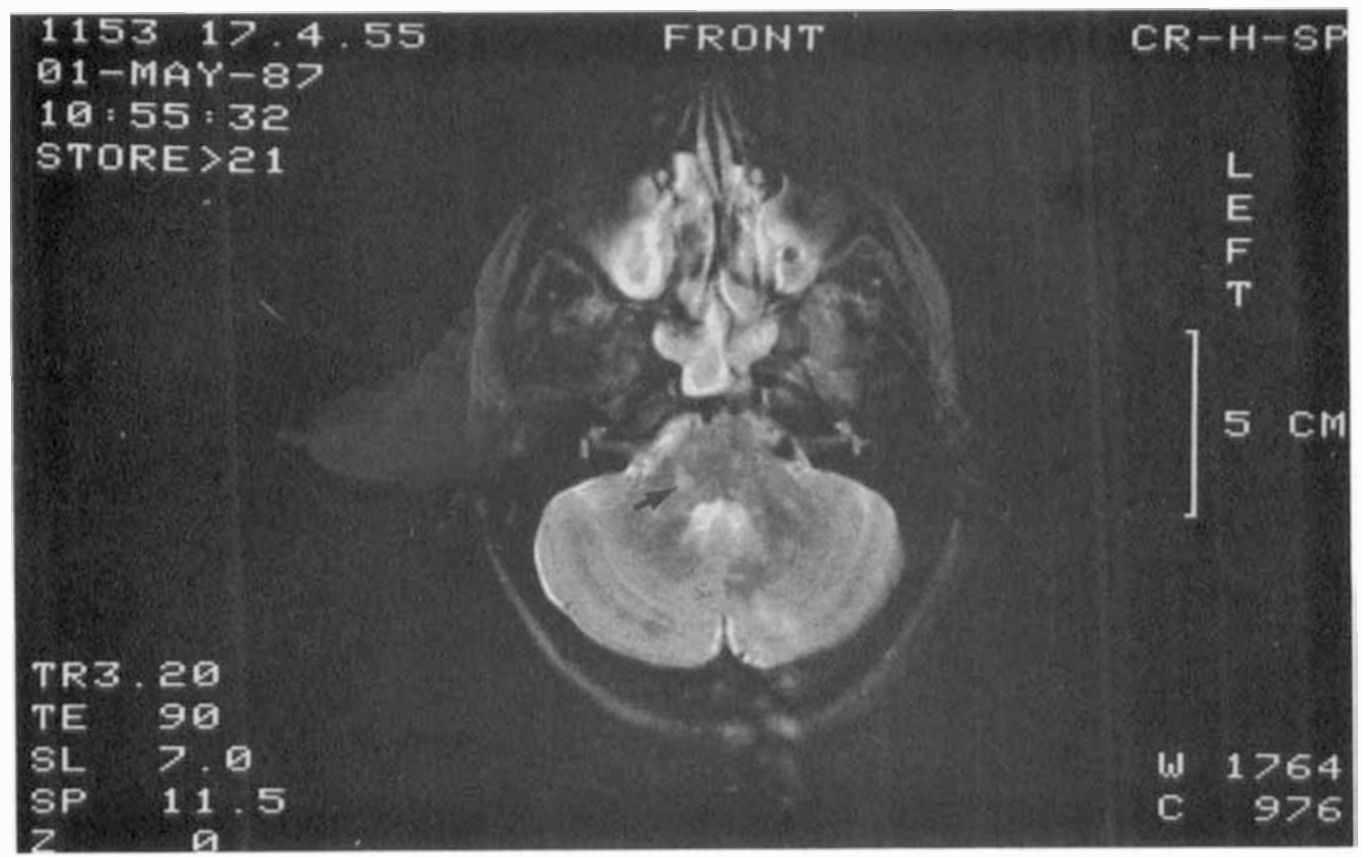

Fig. 7. Case 4. Scattered high intensity lesions are seen on a T2 weighted scan in the pons and cerebellum of a patient with multiple sclerosis who presented with a right VI palsy. The causative lesion is arrowed.

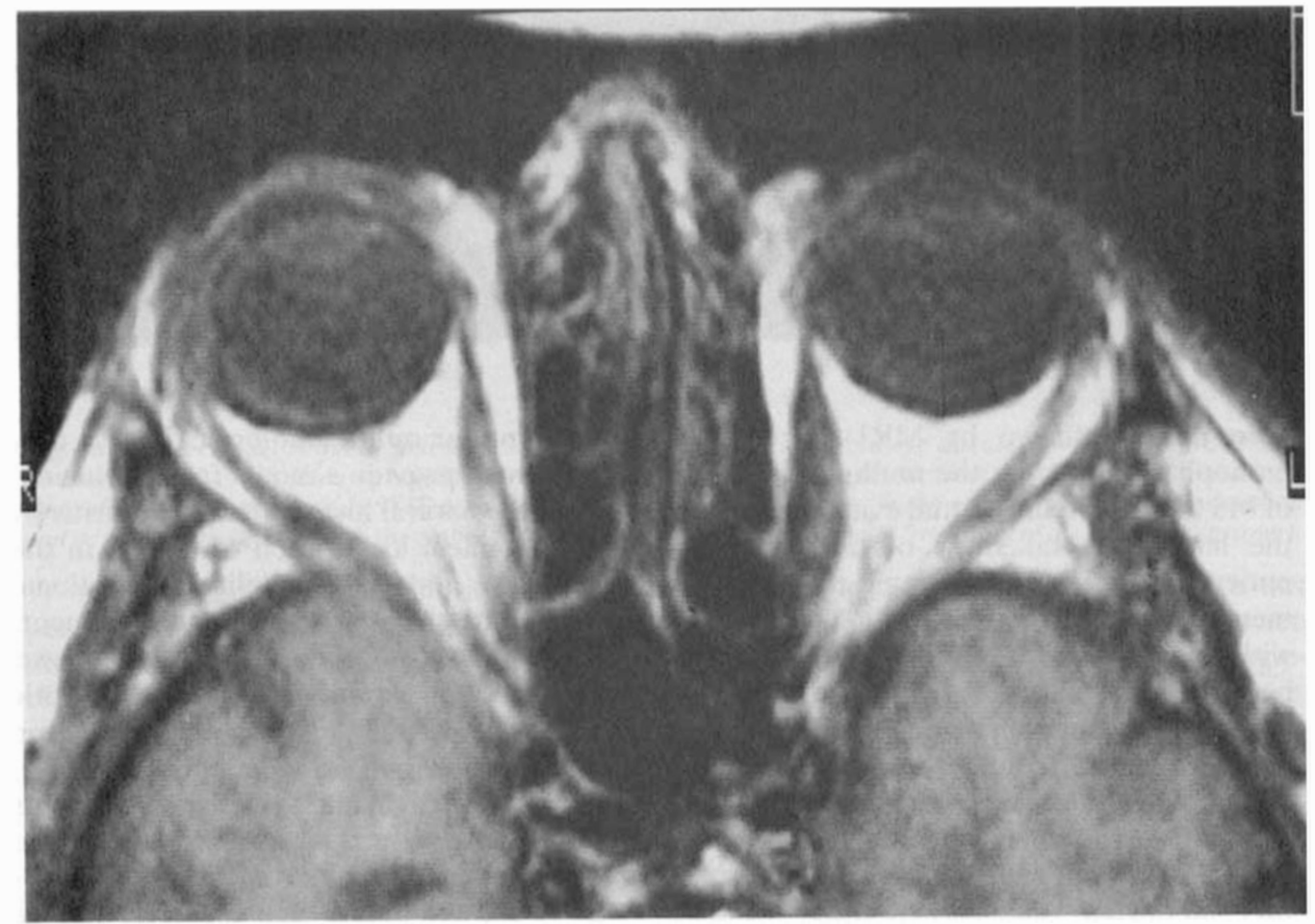

Fig. 8a 


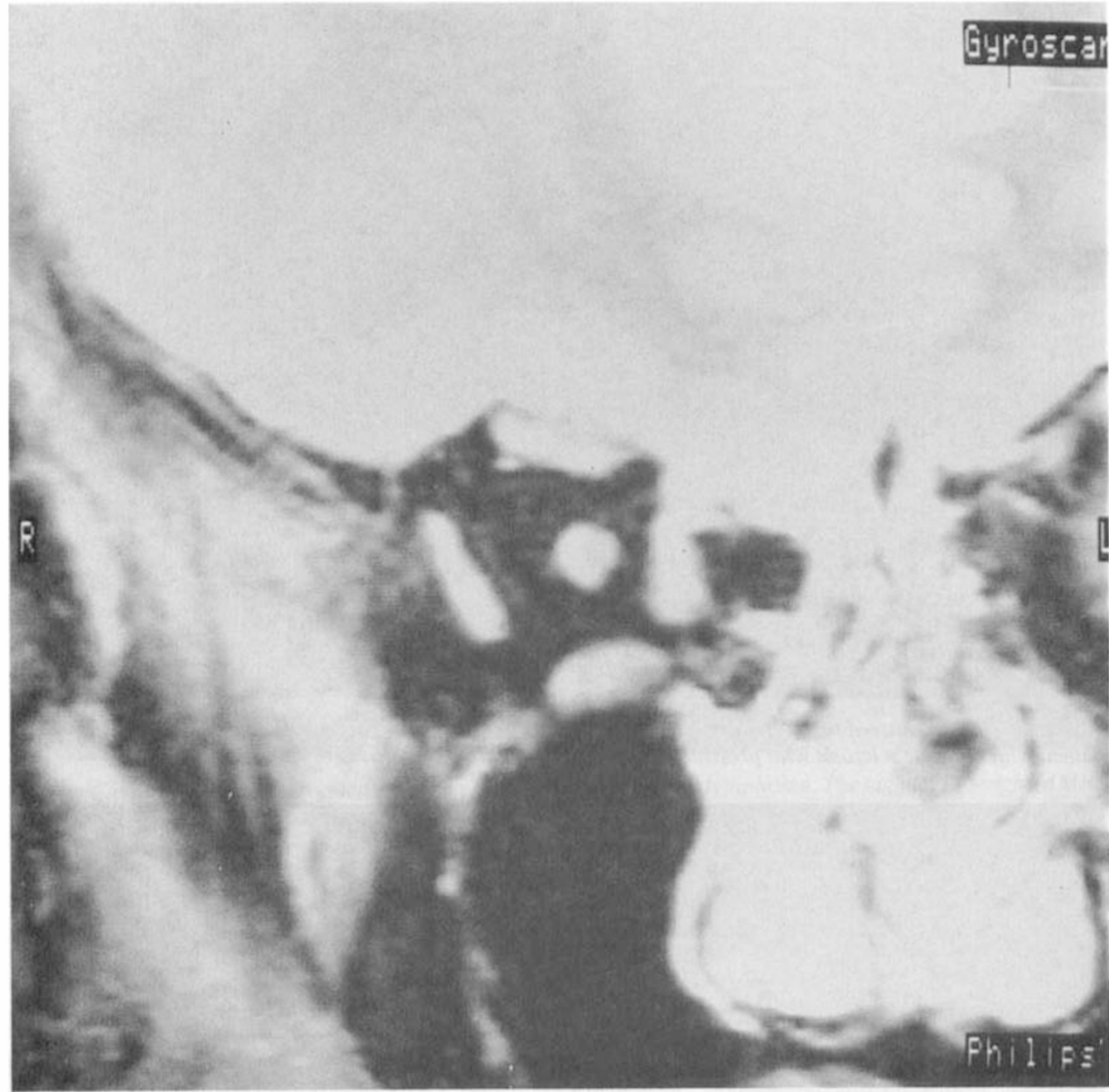

Fig. 8b

these lesions shown by MRI represent a monophasic illness or the multiphasic illness of MS but the circumstantial evidence favours the latter. In one study of this group of patients MRI lesions predicted the development of MS in $95 \%$ of those who developed it within one year of follow up. Correlation between MRI lesions and CSF findings is not tight: patients may have positive MRI scans and negative CSF or vice versa but the combination of both tests being positive produces a $95 \%$ certainty of a diagnosis of clinically definite MS. This leads to the concept of a 'laboratory supported' diagnosis of MS. ${ }^{6,7}$ Studies such as those of Miller et al. are particularly interesting for ophthalmologists in that they are likely to provide a model for the elucidation of the natural history of optic neuritis by the anatomical localisation of lesions in the optic nerve and the possibility of a rational approach to visual prognosis and treatment. In this study a poorer visual prognosis was associated with extensive plaques in the optic nerve or when plaques were situated within the optic canal.

Generally MRI units with larger magnets give clearer and more detailed images than those with smaller magnets. Pituitary adenomas of greater than $3 \mathrm{~mm}$ diameter can be detected by 1.5 Tesla scanners. In a study of 


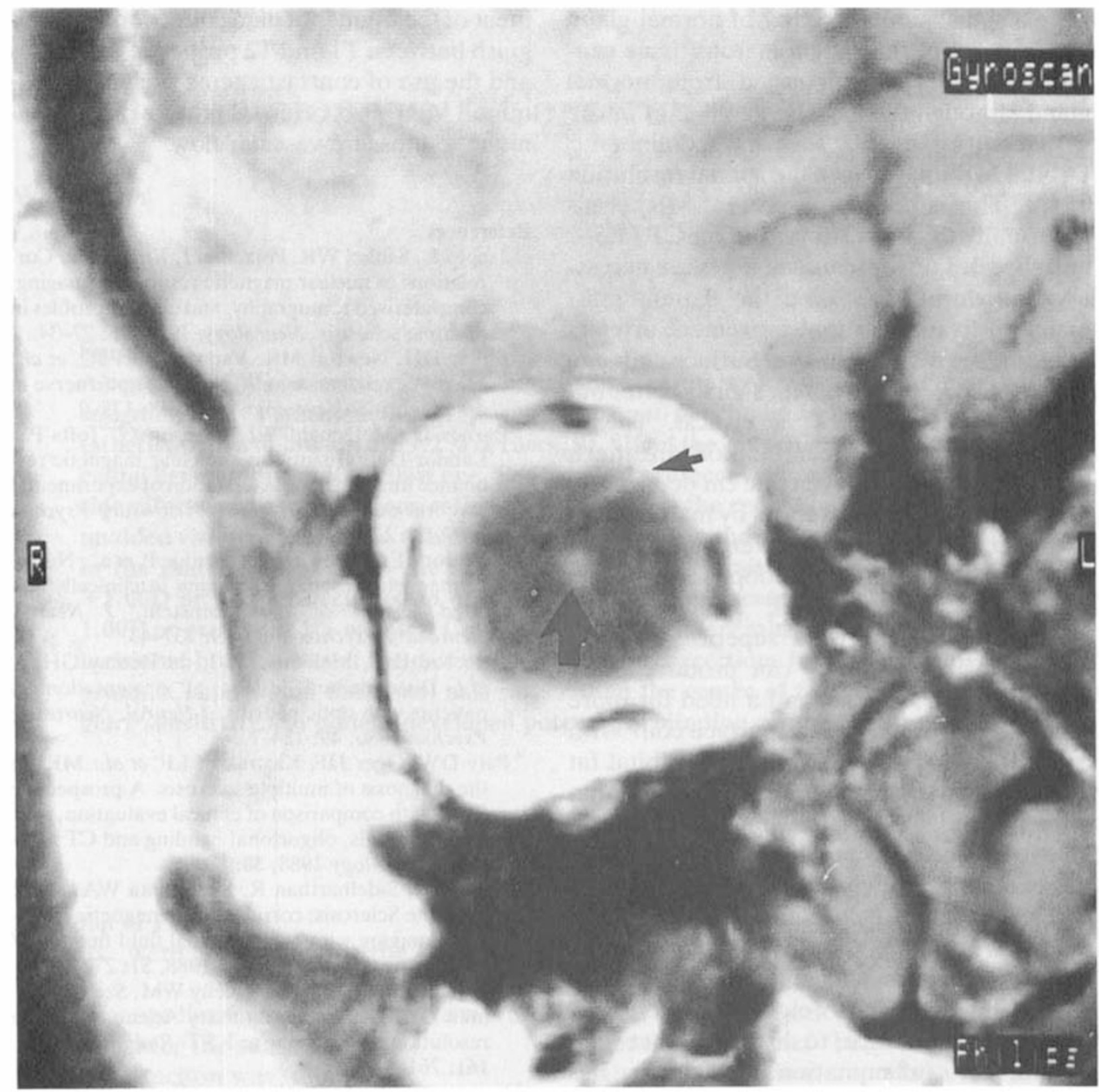

Fig. 8c

Fig. 8a, b and c. 8a. Axial orbital MRI scan of a patient with dysthyroid eye disease. The orbital muscles are visualised but the high intensity signal from the orbital fat detracts from detail. Note that bone is not visualised. Specialised techniques such as STIR sequences give better quality pictures. $8 b$ shows a coronal mid orbital section and $8 c$ shows a section through the globe. Note the vortex veins and high signal from lipid in the optic disc (arrows).

17 macroadenomas excellent imaging was performed in all cases ${ }^{8}$ using $\mathrm{T} 1$ weighted images. The optic nerves and chiasm and their relationship to the tumour can be visualised and haemorrhage can be differentiated from cystic changes more easily than by CT scanning. An interesting feature of MRI is that the carotid arteries are easily visualised and the degree of infiltration of the cavernous sinus assessed more easily than by CT scanning where both vascular spaces enhance with con- trast making differentiation difficult. In this respect, a helpful differentiating sign between pituitary adenomas or suprasellar meningiomas is that meningiomas tend to produce carotid artery constriction or obstruction whereas this is not a feature of adenomatous tissue. In the normal patient the anterior and posterior lobes of the pituitary gland can be differentiated by the higher $\mathrm{T} 1$ signal of the posterior lobe. ${ }^{9}$ This distinction cannot be made by CT scanning. Adenomas tend to 
have a signal similar to that of normal gland and because of this, adenomatous tissue cannot usually be distinguished from normal gland although this may be possible in future with contrast agents such as gadolinium. ${ }^{10}$ Orbital MR images lack the spatial resolution of CT. This is because at present MRI scans are usually thicker $(3-5 \mathrm{~mm})$ than CT (1.5-2 $\mathrm{mm}$ slices). Longer scanning times are necessary and during this time the patient must maintain fixation so that movement artefact does not degrade the image. Surface coils can be used to detect the weaker signals better but the placement of these is critical. This is because the signals near the coil are much stronger than signals even one $\mathrm{cm}$ deeper and it is easy to generate artefacts by malpositioning. Quality images depend very much on the expertise of the staff and cooperation of the patient.For orbital work higher power machines are not always superior to lower power magnets as they can produce more chemical shift artefacts and a need for more accurate positioning of the surface coil. With conventional spin echo sequences orbital fat gives a high signal which obscures other anatomical details. Special techniques are available to overcome some of these problems ${ }^{5}$ but these are not widely available and require special expertise. Nevertheless useful orbital scans can be obtained which add helpful information on tissue diagnosis by looking at changes in $\mathrm{T} 1$ and $\mathrm{T} 2$ weighted images. This can help, for instance, to distinguish between tumour or inflammation. ${ }^{11}$ Melanin has characteristic T1 and T2 times and MRI offers potential in the diagnosis and management of intraocular tumours.

There are many new developments with MRI that are likely to be reflected in clinical practice during the next few years. A major advance should be better tissue diagnosis and this is likely to come with further develop- ment of techniques of data collection to distinguish between $\mathrm{T} 1$ and $\mathrm{T} 2$ properties of tissues and the use of contrast agents such as gadolinium. MRI also offers a non-invasive technique to measure vascular flow.

\section{References}

${ }^{1}$ Jacobs L, Kinkel WR, Polachini I, Kinkel RP: Correlations of nuclear magnetic resonance imaging, computerised tomography, and clinical profiles in multiple sclerosis. Neurology, 1986, 36: 27-34.

${ }^{2}$ Miller DH, Newton MR, Van der Poel JC, et al.: Magnetic resonance imaging of the optic nerve in optic neuritis. Neurology, 1987, 38: 175-9.

${ }^{3}$ Barnes D, McDonald WI, Johnson G, Tofts PS, Landor DN: Quantitative nuclear magnetic resonance imaging, characterisation of experimental cerebral oedema. J Neurol, Neurosurg Psychiat 1987, 50: 125-33.

${ }^{4}$ Ormerod IEC, Bronstein A, Rudge P, et al.: Nuclear magnetic resonance imaging in clinically isolated lesions of the brainstem. J Neurol, Neurosurg Psychiat 1986, 49: 737-43.

${ }^{5}$ Ormerod IEC, McDonald WI, du Boulay GH, et al.: Disseminated lesions at presentation in patients with optic neuritis. J Neurol, Neurosurg Psychiat 1986, 49: 124-7.

${ }^{6}$ Paty DW, Oger JJF, Kastrakoff LF, et al.: MRI in the diagnosis of multiple sclerosis. A prospective study with comparison of clinical evaluation, evoked potentials, oligoclonal banding and CT scanning. Neurology 1988, 38: 180-5.

${ }^{7}$ Honig LS, Sidelharthan R, Sheremata WA, et al.: Multiple Sclerosis: correlation of magnetic resonance imaging with cerebrospinal fluid findings. $J$ Neurol, Neurosurg Psychiat 1988, 51: 277-80.

${ }^{8}$ Kucharceyk W, Davis DO, Kelly WM, Sze G, Norman D, Newton TH: Pituitary Adenomas. High resolution MR imaging at 1.5 T. Radiology 1986, 161: 761-5.

${ }^{9}$ Fujusawa I, Asato R, Nishimura K, et al.: Anterior and posterior lobes of the pituitary gland assessment by $1.5 \mathrm{~T}$ MR imaging. J Computer Assisted Tomography 1987, 11: 214-20.

${ }^{10}$ Davis PC, Hoffman JC, Spencer T, et al : : MR Imaging of Pituitary Adenoma, CT clinical and surgical correlation. Am J Roentgenol 1987, 148: 797-802.

${ }^{11}$ Atlas SW, Grossman RI, Savino PI, et al.: Surface coil MR of orbital pseudotumour. Am J Roentgenol 1987, 148: 803-8. 